\title{
Numerical Studies on the Effects of Variable Viscosity and Thermal Conductivity with Joules Dissipation on the MHD Micropolar Fluid Flow, Heat and Mass Transfer Past a Stretching Porous Surface Embedded in a Porous Medium
}

\author{
Surajit Dutta ${ }^{1}$, Gopal Chandra Hazarika ${ }^{2}$, \\ ${ }^{I}$ Department of Mathematics, C N B College, Bokakhat, Golaghat, Assam, India \\ ${ }^{2}$ Department of Mathematics, Dibrugarh University, Dibrugarh, Dibrugarh, Assam, India
}

*Corresponding Author: Surajit Dutta, Department of Mathematics, C N B College, Bokakhat, Golaghat, Assam. India

\begin{abstract}
The effects of variable viscosity and thermal conductivity on the MHD (Magnetohydrodynamics) fluid flow, heat and mass transfer past a stretching porous surface embedded in a porous medium are investigated numerically taking both viscous and Joules dissipation in to account. The micro-inertia density is considered as variable. Viscosity and thermal conductivity are assumed to be inverse linear function of temperature. Using similarity transformations partial differential equations are converted to ordinary one and the system is then solved using Runge-Kutta method. The effects of various parameters in the problem are shown graphically and skin-friction coefficient and rate of heat transfer are shown in tabular form.
\end{abstract}

Keywords: Micropolar fluid, variable viscosity and thermal conductivity, MHD Flow, porous medium, Joules dissipation, viscous dissipation.

\section{INTRODUCTION}

In the mid of 1960, A.C. Eringen [1-3] introduce micropolar fluid theory. As the Navier-Stokes equations fails to describe the behavior of Newtonian fluids with suspended particles, Eringen introduce the concept of micropolar fluid. It is assumed to be illustrated by two independent vectors, the classical velocity and the micro-rotation vector. As well as its usual rigid motion, fluid points contained in a small volume element, can rotate about the centroid of the volume element. Liquid crystals, animal blood, other polymeric fluids, fluids containing minute amount additives, clouds with dust etc are some examples of micropolar fluids.

The problem of MHD flow and heat transfer over a stretching surface is important due to its applications in industry. For example, in the extrusion of a polymer sheet, the properties of the final product considerably depend on the rate of cooling. During the last few decades so many investigations have made. Stagnation flow of micropolar fluids with strong and weak interaction was studied by Guram G.S. et al. [4]. Ishak A. et al. [5] investigated the heat transfer over a stretching surface with variable heat flux in micropolar fluids. Effects of variable viscosity and thermal conductivity on the MHD flow of micropolar fluid past an accelerated infinite vertical insulated plate was studied by Thakur P.M. et al. [6].

Due to several applications like chemical industry, cooling of nuclear reactors etc. the study of heat transfer and the flow of a viscous fluid over a stretching surface have motivated considerable interest. In the presence of non-uniform heat source effects of thermal buoyancy and variable thermal conductivity in a power law fluid past a vertical stretching sheet was studied by Abel M.S. et al. [7].Effects of viscous dissipation on heat transfer in a non-Newtonian liquid film over an unsteady stretching sheet was studied by Chen C.H. [8]. Hazarika G.C. et al. [9] investigated the effects of variable viscosity and thermal conductivity on MHD mixed convective flow over a stretching surface with radiation. Kelson N.A. et al. [10] studied the effects of surface conditions on flow of micropolar fluid driven by a porous stretching sheet. The effects of variable viscosity and thermal conductivity on liquid film on an unsteady stretching surface was studied by Khound P.K. et al. [11]. Convective heat 
Numerical Studies on the Effects of Variable Viscosity and Thermal Conductivity with Joules Dissipation on the MHD Micropolar Fluid Flow, Heat and Mass Transfer Past a Stretching Porous Surface Embedded in a Porous Medium

transfer in an electrically conducting fluid at a stretching surface in uniform free stream was studied by Vajravelu K. et al. [12]. Ahmed N. et al. [13] investigated the MHD free and forced convection and mass transfer flow past a porous vertical plate. In the presence of magnetic field Sarma U. et al. [14] investigated the effects of variable viscosity and thermal conductivity on heat and mass transfer flow along a vertical plate. Effects of variable viscosity and thermal conductivity on magnetohydrodynamic free convection flow was investigated by Hazarika G.C. et al. [15] when the fluid past a stretching plate through porous medium with radiation.

The main objective of our present work is to extend the work of Anjali Devi S.P. et al. [16] to study the effects of viscous and Joules dissipation on MHD micropolar fluid flow, heat and mass transfer past a stretching porous surface embedded in a porous medium, considering viscosity and thermal conductivity as inverse linear functions of temperature. The problem is solved numerically using fourth order Runge-Kutta shooting algorithm with iteration process after reducing the partial differential equations to ordinary one using similarity transformations.

\section{Mathematical Formulation of the Problem}

Two-dimensional, nonlinear, steady, MHD laminar boundary layer flow with heat and mass transfer of a viscous, incompressible and electrically conducting micropolar fluid over a porous surface embedded in a porous medium is considered for investigation. A uniform transverse magnetic field of strength $B_{0}$ is applied parallel to y-axis. Both viscous and Joules dissipation are taken into account. Consider a polymer sheet emerging out of a slit at $\mathrm{x}=0, \mathrm{y}=0$ and subsequently being stretched, as in a polymer extrusion process. Let us assume that the speed at a point in the plate is proportional to the power of its distance from the slit and the boundary layer approximations are applicable. Induced magnetic field, the external electric field and the electric field due to the polarization of charges are assumed to be negligible in writing the following equations.

\subsection{Basic Equations}

Equation of continuity

$$
\frac{\partial u}{\partial x}+\frac{\partial v}{\partial y}=0
$$

Momentum equation

$$
u \frac{\partial u}{\partial x}+v \frac{\partial u}{\partial y}=\frac{\partial}{\partial y}\left(v \frac{\partial u}{\partial y}\right)+\frac{\kappa}{\rho}\left(\frac{\partial N}{\partial y}+\frac{\partial^{2} u}{\partial y^{2}}\right)-\frac{\sigma B_{0}{ }^{2}}{\rho} u-\frac{v}{K_{p}} u
$$

Angular momentum equation

$$
u \frac{\partial N}{\partial x}+v \frac{\partial N}{\partial y}=\frac{\gamma}{\rho j} \frac{\partial^{2} N}{\partial y^{2}}-\frac{\kappa}{\rho j}\left(2 N+\frac{\partial u}{\partial y}\right)
$$

Energy equation

$$
u \frac{\partial T}{\partial x}+v \frac{\partial T}{\partial y}=\frac{1}{\rho c_{\mathrm{p}}} \frac{\partial}{\partial y}\left(\lambda \frac{\partial T}{\partial y}\right)+\frac{v}{c_{\mathrm{p}}}\left(\frac{\partial u}{\partial y}\right)^{2}+\frac{\sigma B_{0}{ }^{2}}{\rho c_{\mathrm{p}}} u^{2}
$$

Species continuity equation

$$
u \frac{\partial c}{\partial x}+v \frac{\partial c}{\partial y}=\frac{1}{\mathrm{~S}_{\mathrm{c}}} \frac{\partial}{\partial y}\left(v \frac{\partial c}{\partial y}\right)
$$

The boundary conditions are

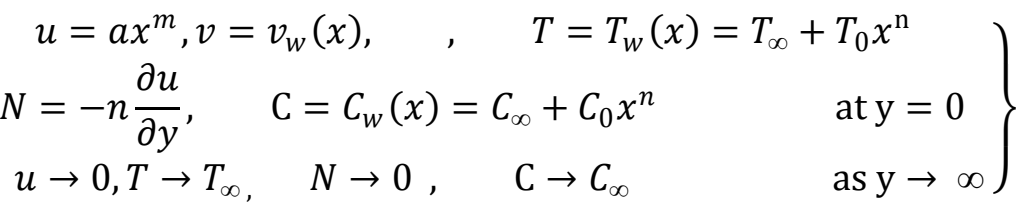


Numerical Studies on the Effects of Variable Viscosity and Thermal Conductivity with Joules Dissipation on the MHD Micropolar Fluid Flow, Heat and Mass Transfer Past a Stretching Porous Surface Embedded in a Porous Medium

Assume the viscosity as inverse linear functions of temperature, as done by Lai and Kulacki [17], i.e.

$$
\begin{gathered}
\frac{1}{\mu}=\frac{1}{\mu_{\infty}}\left[1+\delta\left(T-T_{\infty}\right)\right], \text { or } \frac{1}{\mu}=a\left(T-T_{r}\right) \\
\text { where } a=\frac{\delta}{\mu_{\infty}} \text { and } T_{r}=T_{\infty}-\frac{1}{\delta}
\end{gathered}
$$

where $T_{r}$ is transformed reference temperature corresponding to viscosity parameter, $\mu_{\infty}$ is the viscosity of the ambient fluid, $a$ and $T_{\infty}$ are constants whose values are depend upon the reference state and thermal property of the fluid.

Similarly

$$
\begin{gathered}
\frac{1}{\lambda}=\frac{1}{\lambda_{\infty}}\left[1+\xi\left(T-T_{\infty}\right)\right] \text {, or } \frac{1}{\lambda}=b\left(T-T_{c}\right) \\
\text { where } b=\frac{\xi}{\lambda_{\infty}} \text { and } T_{c}=T_{\infty}-\frac{1}{\xi}
\end{gathered}
$$

where $b$ and $T_{c}$ are constants and their values depend on the reference state and thermal properties of the fluid.

Let us introduce the following non-dimensional variables

$$
\left.\begin{array}{c}
\psi(x, y)=\left[\frac{2 v_{\infty} x U_{w}}{1+m}\right]^{1 / 2} f(\eta), \quad \eta=\left[\frac{(1+m) U_{w}}{2 v_{\infty} x}\right]^{1 / 2} y, \quad N=\left(\frac{(m+1) U_{w}^{3}}{2 v_{\infty} x}\right)^{\frac{1}{2}} h(\eta), \\
j=\frac{v_{\infty} x}{(m+1) U_{w}} i(\eta), \quad v_{w}(x)=-\lambda_{0}\left\{\frac{v_{\infty} a(1+m)}{2}\right\}^{1 / 2} x^{\frac{m-1}{2}}
\end{array}\right\}
$$

and non-dimensional form of temperature and the concentration as

$$
\theta=\frac{T-T_{\infty}}{T_{w}-T_{\infty}}, g=\frac{C-C_{\infty}}{C_{w}-C_{\infty}}
$$

where $\lambda_{0}>0$ for suction at the stretching plate and $\psi$ is the stream function.

The velocity components are given by $u=\frac{\partial \psi}{\partial y}, v=-\frac{\partial \psi}{\partial x}$.

The equation of continuity (1) is identically satisfied. Substituting above transformations in equations (2) - (5), we get

$$
\begin{aligned}
& \left(\frac{\theta_{r}}{\theta-\theta_{r}}-K\right) f^{\prime \prime \prime}=f f^{\prime \prime}-\frac{2 m}{m+1} f^{\prime 2}+\frac{\theta_{r}}{\left(\theta-\theta_{r}\right)^{2}} f^{\prime \prime} \theta^{\prime}+\frac{1}{R_{1}} \frac{\theta_{r}}{\theta-\theta_{r}} f^{\prime}+K h^{\prime}-M f^{\prime} \\
& \left(1+\frac{1}{2} K\right) h^{\prime \prime}=2\left(2 h+f^{\prime \prime}\right) K_{1}+\frac{3 m-1}{m+1} h f^{\prime}-h^{\prime} f \\
& \frac{\theta_{c}}{\theta-\theta_{c}} \theta^{\prime \prime}=\frac{\theta_{c}}{\left(\theta-\theta_{c}\right)^{2}} \theta^{\prime 2}+P_{r} f \theta^{\prime}-\frac{\theta_{r}}{\theta-\theta_{r}} E_{c} P_{r} f^{\prime \prime 2}+E_{c} P_{r} M f^{\prime 2} \\
& g^{\prime \prime}=\frac{1}{\theta-\theta_{r}} \theta^{\prime} g^{\prime}+S_{c} \frac{\theta-\theta_{r}}{\theta_{r}} g^{\prime} f
\end{aligned}
$$

and the boundary conditions (6)become

$$
\left.\begin{array}{c}
\eta=0: f=\lambda_{0}, f^{\prime}=1, g=1, \theta=1, h=-n f^{\prime \prime} \\
\eta \rightarrow \infty: f^{\prime}=0, \theta=0, g=0, h=0
\end{array}\right\}
$$


Numerical Studies on the Effects of Variable Viscosity and Thermal Conductivity with Joules Dissipation on the MHD Micropolar Fluid Flow, Heat and Mass Transfer Past a Stretching Porous Surface Embedded in a Porous Medium

The important physical quantities of this problem are the skin friction coefficient $c_{f}$ and local Nusselt number $N u_{x}$ which indicates physically the rate of surface shear stress and rate of heat transfer respectively.

The shear stress is given by

$$
\tau_{w}=\left[(\mu+\kappa) \frac{\partial u}{\partial y}+\kappa N\right]_{y=0}
$$

The skin friction coefficient $c_{f}$ is defined as

$$
c_{f}=\frac{2 \tau_{w}}{\rho U_{w}^{2}}
$$

The rate of heat transfer from the surface is given by

$$
\begin{gathered}
q_{w}=-\lambda\left[\frac{\partial T}{\partial y}\right]_{y=0} \\
N u_{x}=\left(\frac{m+1}{2}\right)^{1 / 2} \frac{\theta_{c}}{1-\theta_{c}}\left(R e_{x}\right)^{1 / 2} \theta^{\prime}(0)
\end{gathered}
$$

where $R e_{x}=U_{w} x / v_{\infty}$ is the local Reynolds number.

\section{RESULTS AND DISCUSSION}

The system of ordinary differential equations (11) - (14) together with the boundary conditions (15) are solved numerically by using the Runge-Kutta fourth order shooting method. The numerical values of different parameters are taken as $M=1, \operatorname{Pr}=0.7, \theta_{c}=4, \theta_{r}=3, K=1, m=1, R_{1}=10, K_{1}=$ $0.5, E_{c}=0.05, S_{c}=0.22$ unless otherwise stated.

The variation in velocity distribution, angular velocity distribution, temperature distribution and species concentration distribution are illustrated in Figs. (1) - (13) with the variation of different parameters.

Figs. (1) - (3) represents the variations in dimensionless velocity distribution with viscosity parameter $\theta_{r}$, magnetic parameter $M$ and coupling constant parameter $K$. From Fig. (1) it is clear that the velocity decreases with the increase of viscosity parameter $\theta_{r}$. From Fig. (2) it is observed that the velocity decreases due to increase of magnetic parameter $M$. Application of magnetic field forms a resistive force (Lorentz force) like drag force, retards the velocity. From Fig. (3) we observe that the velocity increase for the increase of coupling constant parameter $K$. It is due to the fact that viscosity decreases with the increase of coupling constant parameter $K$ and as a result velocity increase.

Figs. (4) - (7) represents the variations in dimensionless angular velocity distribution for various values of coupling constant parameter $K_{1}$ and $\mathrm{K}$, magnetic parameter $M$ and viscosity parameter $\theta_{r}$. We observe that angular velocity increase for the increase of all these parameters $K_{1}, K, M$ and $\theta_{r}$.

Figs. (8) - (10) represents the dimensionless temperature profile $\theta(\eta)$ with the variation of viscosity parameter $\theta_{r}$, thermal conductivity parameter $\theta_{c}$ and magnetic parameter $M$. Figs. (8) and (10) shows that temperature increase for the increase of viscosity parameter $\theta_{r}$ and magnetic parameter $M$. As in both cases a resistive force will act against the fluid flow and as a result temperature will increase. From Fig. (9) we observe that the temperature decrease for the increase of the thermal conductivity parameter $\theta_{c}$.

Figs. (11) - (13) shows the distribution representing species concentration profile within the boundary layer with the variation of $\theta_{r}, S_{c}$ and $M$. Figs. (11) and (12) shows that concentration decreases with the increase of $\theta_{r}$ and $S_{c}$. $S_{c}$ connects velocity and concentration profiles. So when $S_{c}$ increases, molecular mass diffusivity decreases resulting the decreasing of concentration. Fig. 13 shows that the concentration increases with the increasing values of $M$. 
Numerical Studies on the Effects of Variable Viscosity and Thermal Conductivity with Joules Dissipation on the MHD Micropolar Fluid Flow, Heat and Mass Transfer Past a Stretching Porous Surface Embedded in a Porous Medium

\subsection{Figures}

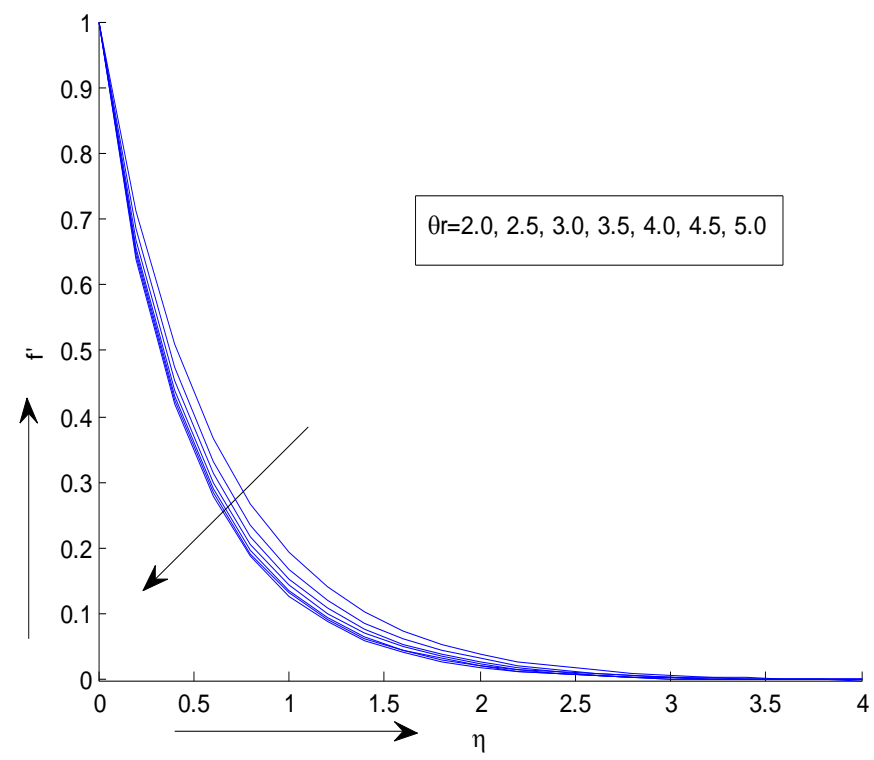

Figure 1. Velocity profile for various $\theta_{r}$.

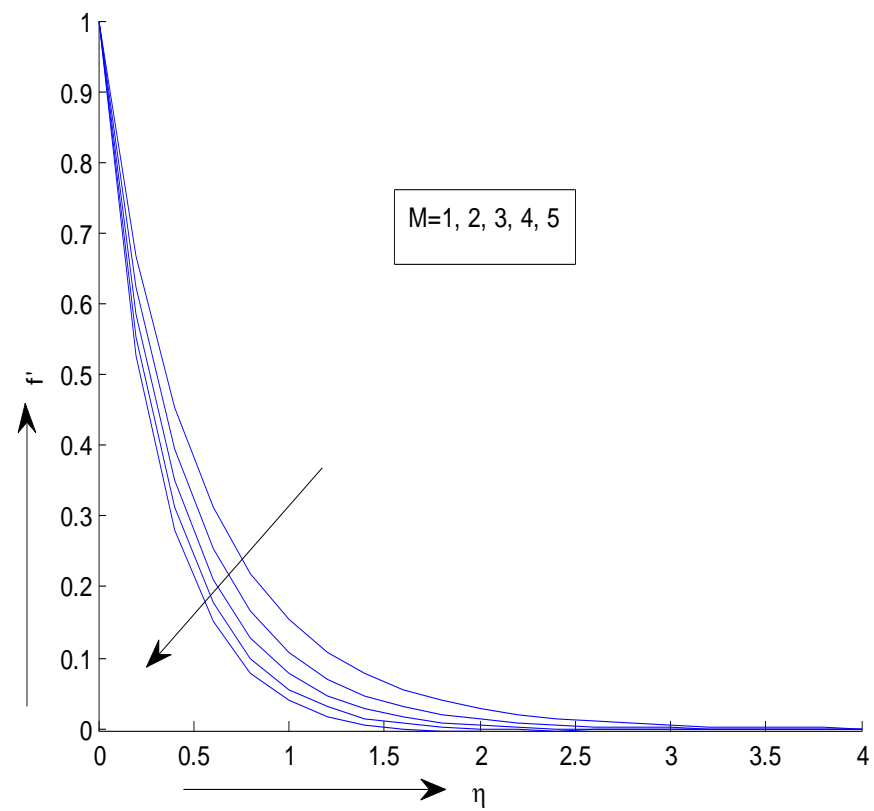

Figure 2. Velocity profile for various $M$ 
Numerical Studies on the Effects of Variable Viscosity and Thermal Conductivity with Joules Dissipation on the MHD Micropolar Fluid Flow, Heat and Mass Transfer Past a Stretching Porous Surface Embedded in a Porous Medium

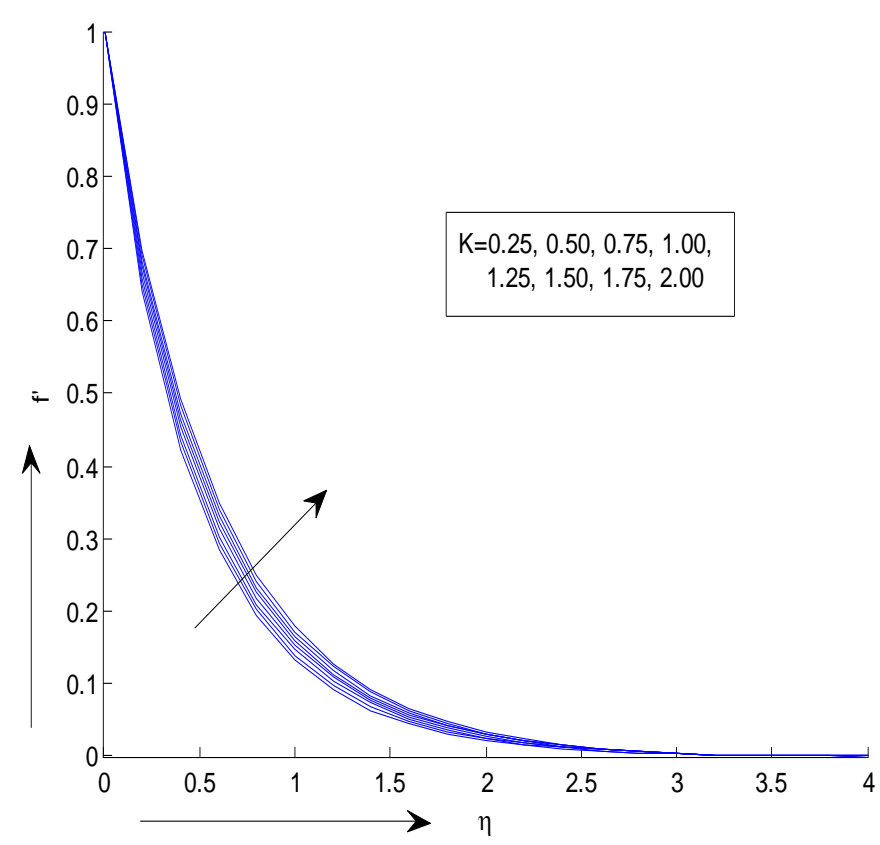

Figure 3. Velocity profile for various $K$.

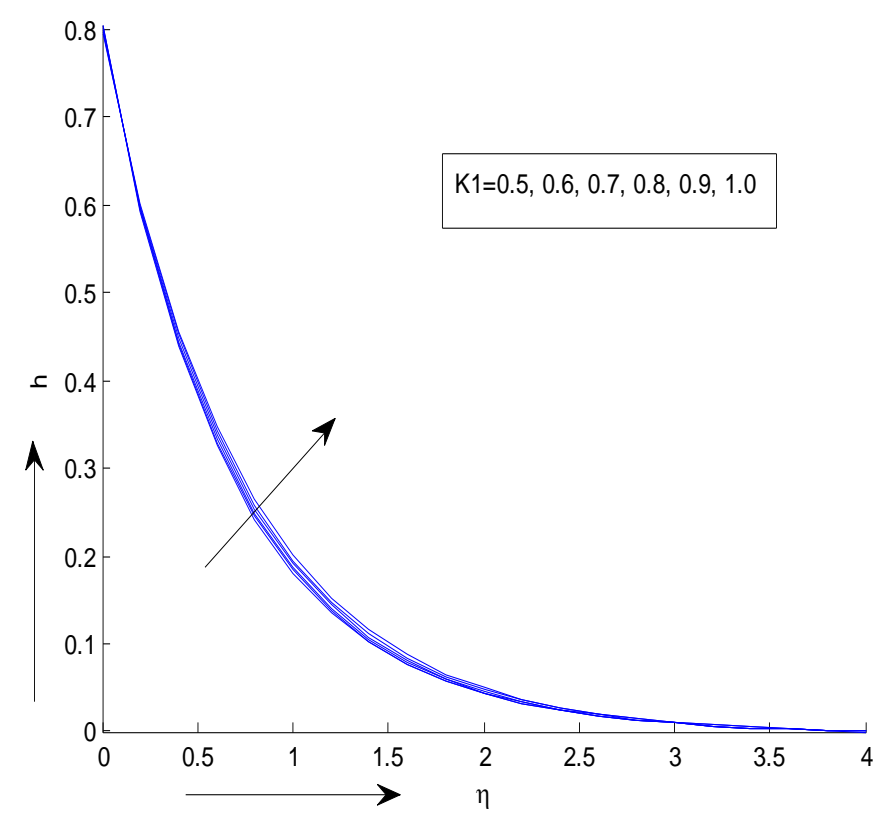

Figure 4. Angular velocity profile for various $K_{1}$. 
Numerical Studies on the Effects of Variable Viscosity and Thermal Conductivity with Joules Dissipation on the MHD Micropolar Fluid Flow, Heat and Mass Transfer Past a Stretching Porous Surface Embedded in a Porous Medium

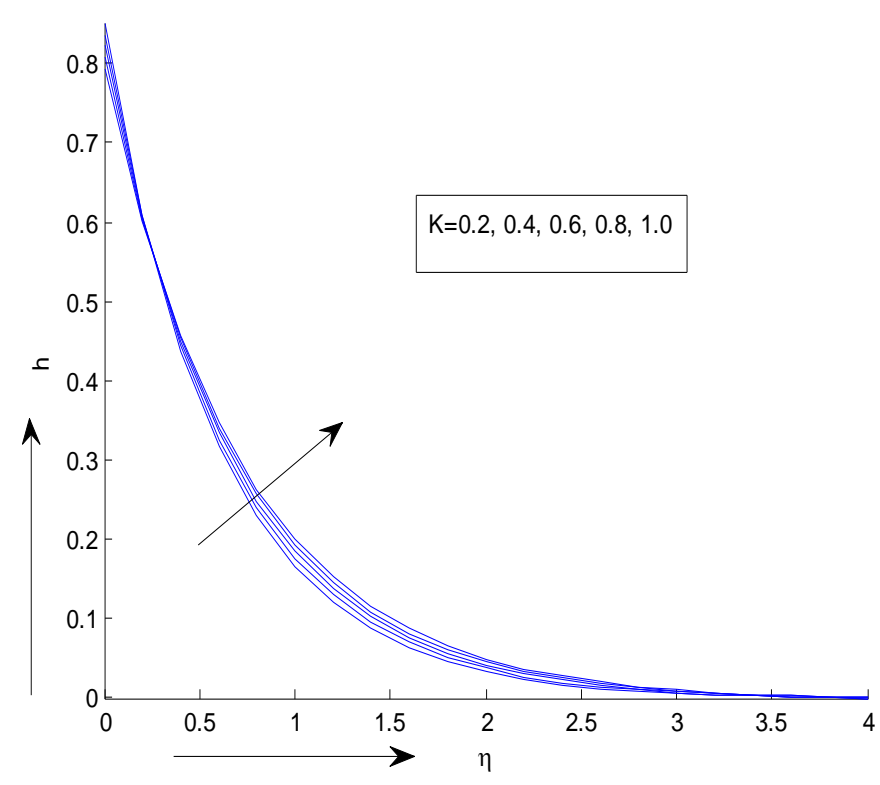

Figure 5. Angular velocity profile for various $K$.

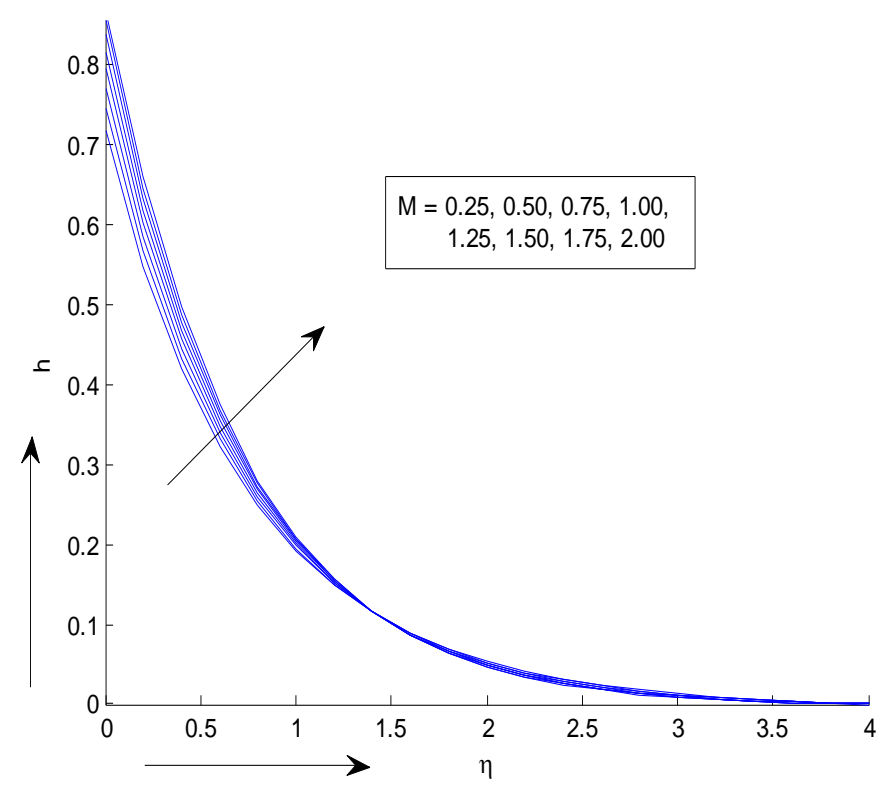

Figure 6. Angular velocity profile for various $M$. 

on the MHD Micropolar Fluid Flow, Heat and Mass Transfer Past a Stretching Porous Surface Embedded in a Porous Medium

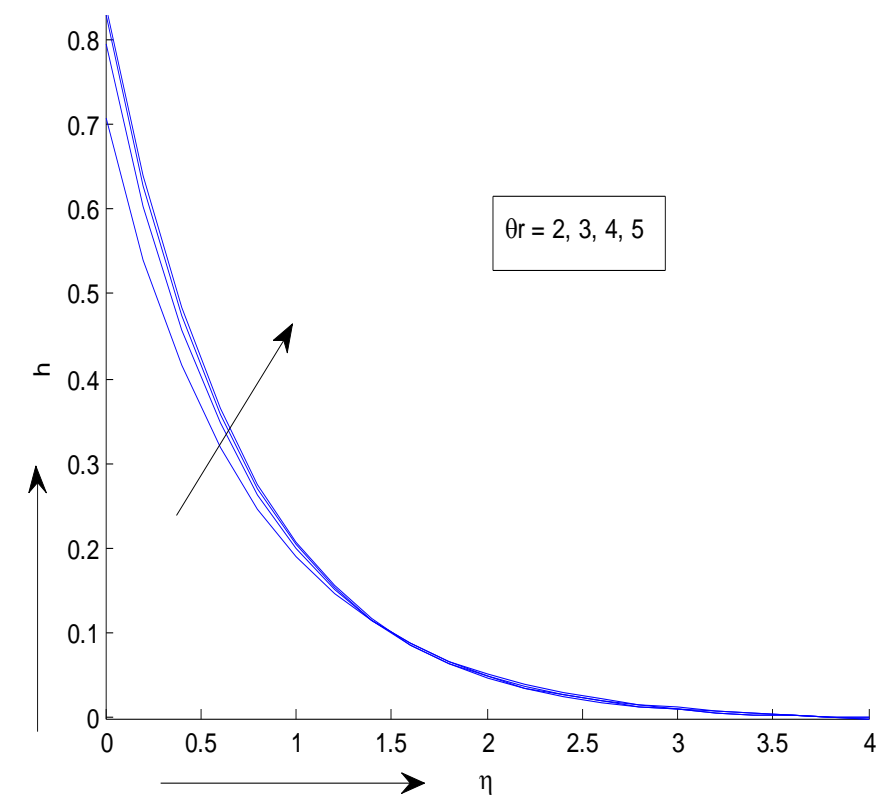

Figure 7. Angular velocity profile for various $\theta_{r}$.

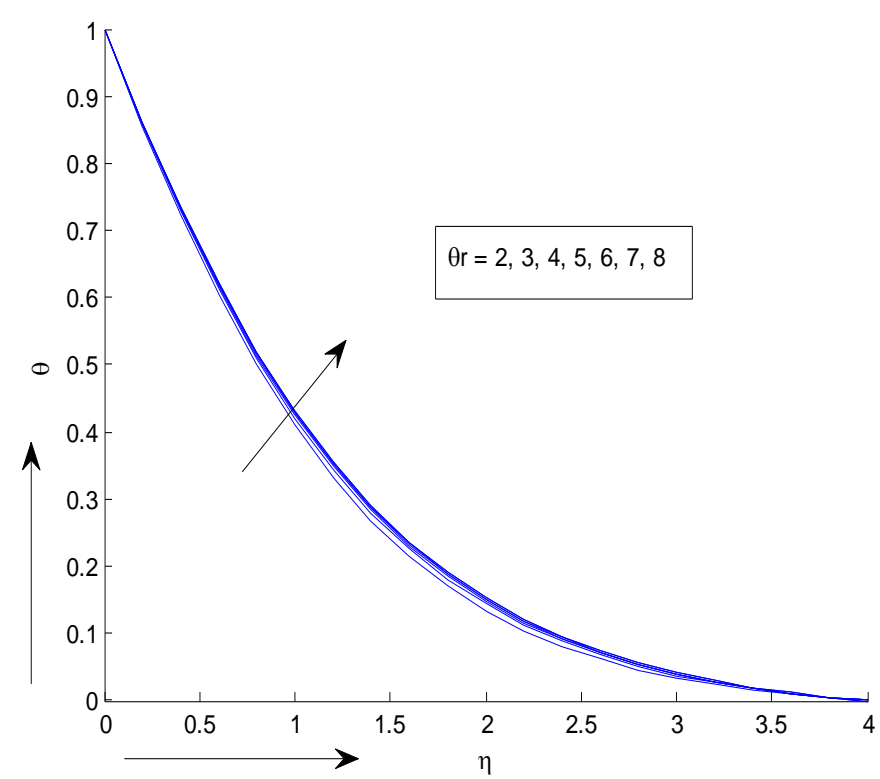

Figure 8. Temperature profile for various $\theta_{r}$. 
Numerical Studies on the Effects of Variable Viscosity and Thermal Conductivity with Joules Dissipation on the MHD Micropolar Fluid Flow, Heat and Mass Transfer Past a Stretching Porous Surface Embedded in a Porous Medium

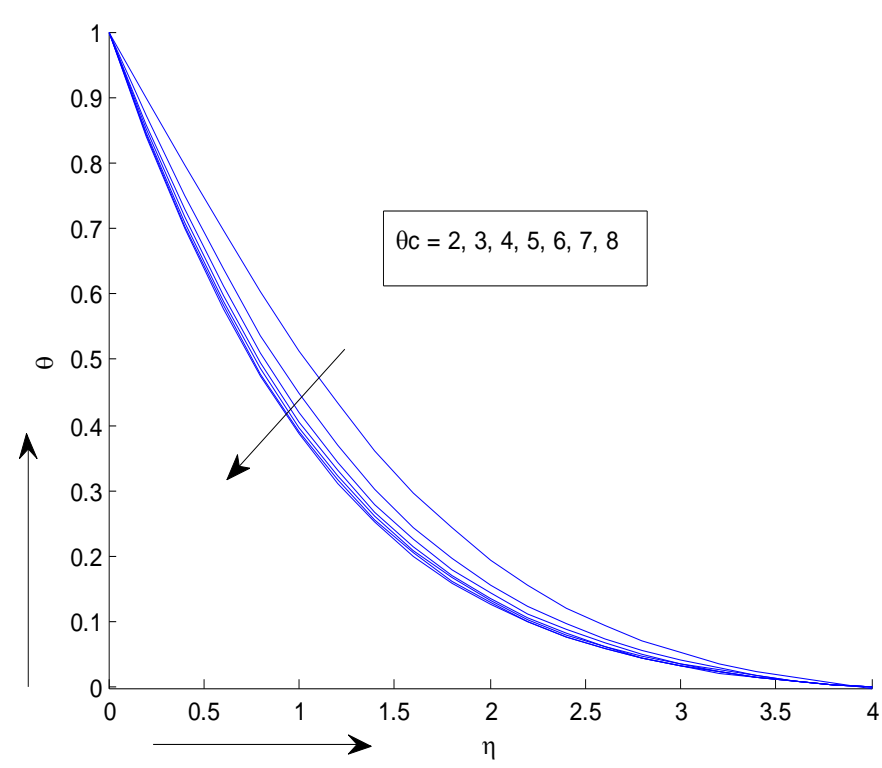

Figure 9. Temperature profile for various $\theta_{c}$.

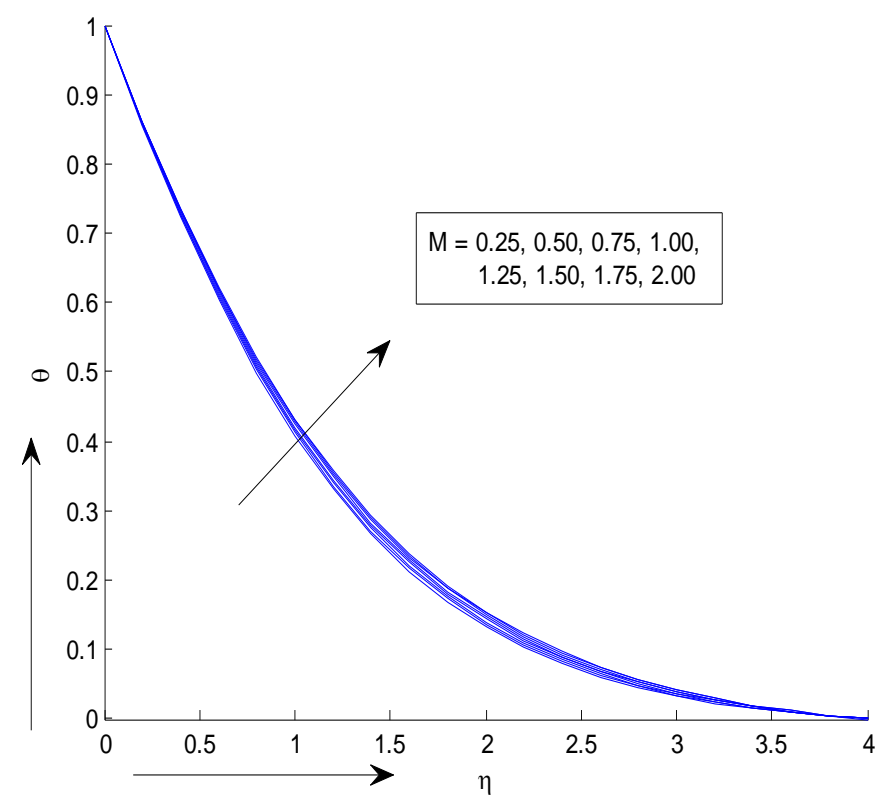

Figure 10. Temperature profile for various $M$. 
Numerical Studies on the Effects of Variable Viscosity and Thermal Conductivity with Joules Dissipation on the MHD Micropolar Fluid Flow, Heat and Mass Transfer Past a Stretching Porous Surface Embedded in a Porous Medium

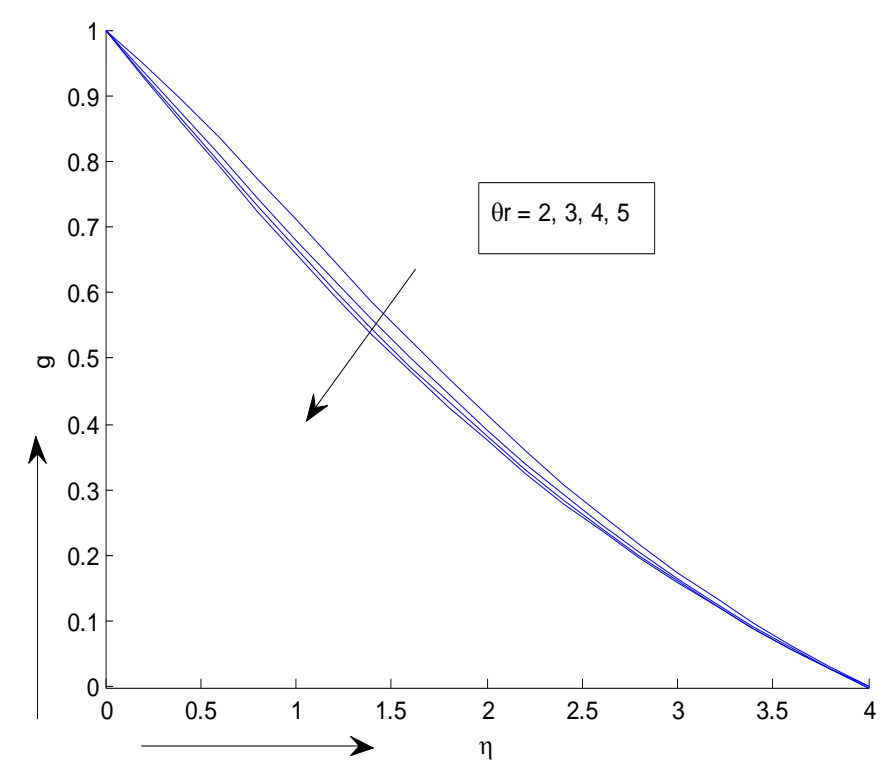

Figure 11. Concentration profile for various $\theta_{r}$.

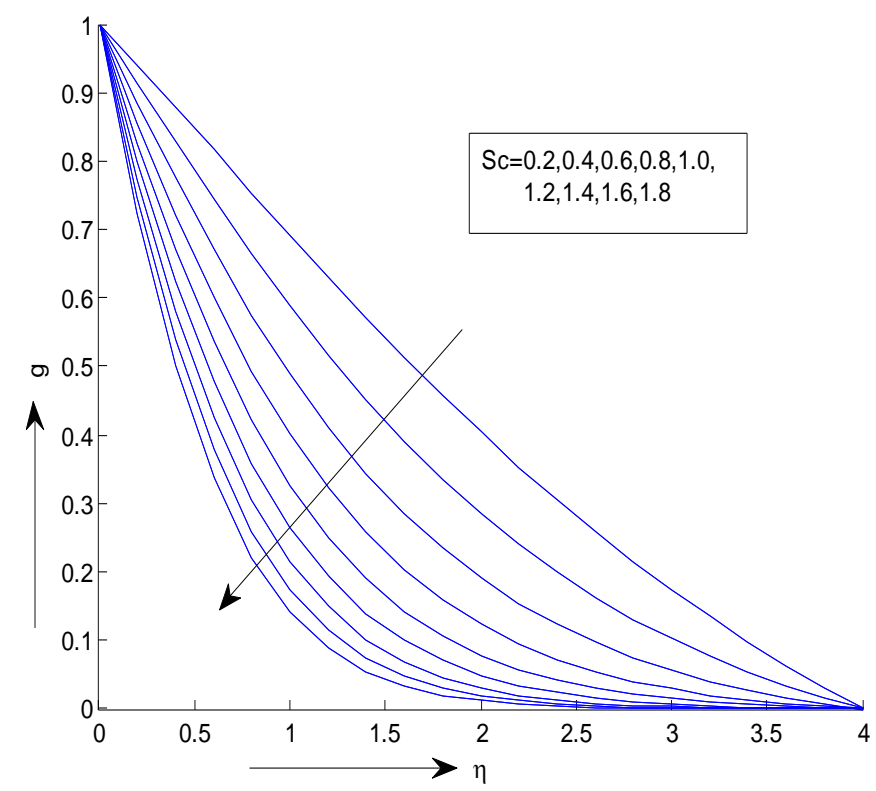

Figure 12. Concentration profile for various $S_{c}$. 
Numerical Studies on the Effects of Variable Viscosity and Thermal Conductivity with Joules Dissipation on the MHD Micropolar Fluid Flow, Heat and Mass Transfer Past a Stretching Porous Surface Embedded in a Porous Medium

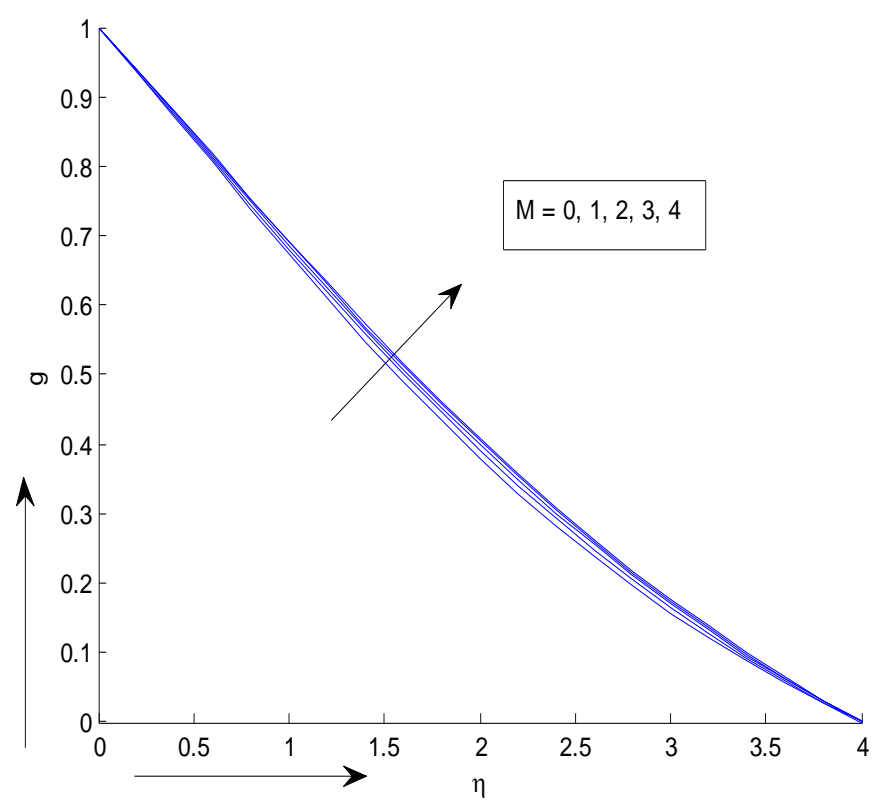

Figure 13. Concentration profile for various $M$.

\subsection{Tables}

Table 1. Missing values and physical quantities with the variation of $M$ and $\theta_{c}$.

\begin{tabular}{|l|c|c|c|c|c|c|c|}
\hline$\theta_{\mathrm{c}}$ & $\mathrm{M}$ & $\mathrm{c}_{\mathrm{f}}$ & $\mathrm{Nu}$ & $f^{\prime \prime}(0)$ & $\theta^{\prime}(0)$ & $h^{\prime}(0)$ & $g^{\prime}(0)$ \\
\hline \multirow{4}{*}{2} & 0.50 & 1.498964 & 1.0111 & -0.99931 & -0.50555 & -1.03763 & -0.31086 \\
\cline { 2 - 8 } & 1.00 & 1.596479 & 0.997356 & -1.06432 & -0.49868 & -1.11714 & -0.30784 \\
\cline { 2 - 8 } & 1.50 & 1.681777 & 0.986128 & -1.12118 & -0.49306 & -1.18695 & -0.30541 \\
\cline { 2 - 8 } & 2.00 & 1.7581 & 0.976819 & -1.17207 & -0.48841 & -1.24962 & -0.3034 \\
\hline 3 & 0.50 & 1.493648 & 0.987639 & -0.99577 & -0.65843 & -1.03209 & -0.30871 \\
\cline { 2 - 8 } & 1.00 & 1.590964 & 0.975415 & -1.06064 & -0.65028 & -1.11151 & -0.30566 \\
\cline { 2 - 8 } & 1.50 & 1.676127 & 0.965368 & -1.11742 & -0.64358 & -1.18129 & -0.30322 \\
\cline { 2 - 8 } & 2.00 & 1.752358 & 0.956993 & -1.16824 & -0.638 & -1.24394 & -0.3012 \\
\hline 4 & 0.50 & 1.491313 & 0.978146 & -0.99421 & -0.73361 & -1.02965 & -0.30786 \\
\cline { 2 - 8 } & 1.00 & 1.588522 & 0.966567 & -1.05902 & -0.72493 & -1.10901 & -0.30481 \\
\cline { 2 - 8 } & 1.50 & 1.673607 & 0.957028 & -1.11574 & -0.71777 & -1.17876 & -0.30235 \\
\cline { 2 - 8 } & 2.00 & 1.74978 & 0.949061 & -1.16652 & -0.7118 & -1.24139 & -0.30032 \\
\hline 5 & 0.50 & 1.489996 & 0.972971 & -0.99333 & -0.77838 & -1.02827 & -0.3074 \\
\cline { 2 - 8 } & 1.00 & 1.587139 & 0.961747 & -1.05809 & -0.7694 & -1.10759 & -0.30434 \\
\cline { 2 - 8 } & 1.50 & 1.672175 & 0.952491 & -1.11478 & -0.76199 & -1.17732 & -0.30188 \\
\cline { 2 - 8 } & 2.00 & 1.748311 & 0.944752 & -1.16554 & -0.7558 & -1.23993 & -0.29985 \\
\hline
\end{tabular}

Table 2. Missing values and physical quantities with the variation of $M$ and $\theta_{r}$

\begin{tabular}{|c|c|c|c|c|c|c|c|}
\hline$\theta_{r}$ & $M$ & $c_{f}$ & $\mathrm{Nu}$ & $f^{\prime \prime}(0)$ & $\theta^{\prime}(0)$ & $h^{\prime}(0)$ & $g^{\prime}(0)$ \\
\hline \multirow{4}{*}{2} & 0.50 & 0.879632 & 0.996193 & -0.87963 & -0.74715 & -0.88278 & -0.24116 \\
\cline { 2 - 8 } & 1.00 & 0.941322 & 0.985133 & -0.94132 & -0.73885 & -0.95846 & -0.2388 \\
\cline { 2 - 8 } & 1.50 & 0.995484 & 0.975798 & -0.99548 & -0.73185 & -1.02523 & -0.23688 \\
\cline { 2 - 8 } & 2.00 & 1.044001 & 0.967846 & -1.044 & -0.72588 & -1.08526 & -0.23528 \\
\hline \multirow{4}{*}{3} & 0.50 & 1.491313 & 0.978146 & -0.99421 & -0.73361 & -1.02965 & -0.30786 \\
\cline { 2 - 8 } & 1.00 & 1.588522 & 0.966567 & -1.05902 & -0.72493 & -1.10901 & -0.30481 \\
\cline { 2 - 8 } & 1.50 & 1.673607 & 0.957028 & -1.11574 & -0.71777 & -1.17876 & -0.30235 \\
\hline \multirow{4}{*}{4} & 2.00 & 1.74978 & 0.949061 & -1.16652 & -0.7118 & -1.24139 & -0.30032 \\
\cline { 2 - 8 } & 0.50 & 1.733045 & 0.970531 & -1.03983 & -0.7279 & -1.08732 & -0.33943 \\
\cline { 2 - 8 } & 1.00 & 1.842703 & 0.958862 & -1.10562 & -0.71915 & -1.1678 & -0.33606 \\
\cline { 2 - 8 } & 1.50 & 1.938648 & 0.949348 & -1.16319 & -0.71201 & -1.2385 & -0.33336 \\
\hline
\end{tabular}


Numerical Studies on the Effects of Variable Viscosity and Thermal Conductivity with Joules Dissipation on the MHD Micropolar Fluid Flow, Heat and Mass Transfer Past a Stretching Porous Surface Embedded in a Porous Medium

\begin{tabular}{|c|c|c|c|c|c|c|c|}
\hline \hline \multirow{4}{*}{5} & 0.50 & 1.862685 & 0.966326 & -1.06439 & -0.72474 & -1.11818 & -0.35788 \\
\cline { 2 - 8 } & 1.00 & 1.97866 & 0.954641 & -1.13066 & -0.71598 & -1.19919 & -0.35432 \\
\cline { 2 - 8 } & 1.50 & 2.080135 & 0.945167 & -1.18865 & -0.70888 & -1.27035 & -0.35147 \\
\cline { 2 - 8 } & 2.00 & 2.171076 & 0.937351 & -1.24062 & -0.70301 & -1.33429 & -0.34914 \\
\hline
\end{tabular}

Table 3. Missing values and physical quantities with the variation of Kand $\theta_{c}$.

\begin{tabular}{|c|c|c|c|c|c|c|c|}
\hline$\theta_{c}$ & $\mathrm{~K}$ & $c_{f}$ & $\mathrm{Nu}$ & $f^{\prime \prime}(0)$ & $\theta^{\prime}(0)$ & $h^{\prime}(0)$ & $g^{\prime}(0)$ \\
\hline \multirow{4}{*}{2} & 0.25 & -0.85325 & 0.983003 & -1.13767 & -0.4915 & -1.42877 & -0.30577 \\
\cline { 2 - 8 } & 0.50 & 0 & 0.988099 & -1.11193 & -0.49405 & -1.30757 & -0.3065 \\
\cline { 2 - 8 } & 0.75 & 0.81559 & 0.992844 & -1.08745 & -0.49642 & -1.20497 & -0.30718 \\
\hline \multirow{4}{*}{3} & 1.00 & 1.596479 & 0.997356 & -1.06432 & -0.49868 & -1.11714 & -0.30784 \\
\cline { 2 - 8 } & 0.25 & -0.84954 & 0.961726 & -1.13272 & -0.64115 & -1.41965 & -0.30353 \\
\cline { 2 - 8 } & 0.50 & 0 & 0.966596 & -1.10749 & -0.6444 & -1.29993 & -0.30428 \\
\hline \multirow{4}{*}{4} & 0.75 & 0.812573 & 0.971122 & -1.08343 & -0.64742 & -1.19846 & -0.30499 \\
\cline { 2 - 8 } & 1.00 & 1.590964 & 0.975415 & -1.06064 & -0.65028 & -1.11151 & -0.30566 \\
\cline { 2 - 8 } & 0.25 & -0.84788 & 0.953181 & -1.1305 & -0.71489 & -1.41556 & -0.30264 \\
\cline { 2 - 8 } & 0.50 & 0 & 0.957948 & -1.10551 & -0.71846 & -1.29651 & -0.30341 \\
\hline \multirow{4}{*}{5} & 0.75 & 0.811233 & 0.962374 & -1.08164 & -0.72178 & -1.19556 & -0.30412 \\
\cline { 2 - 8 } & 0.00 & 1.588522 & 0.966567 & -1.05902 & -0.72493 & -1.10901 & -0.30481 \\
\cline { 2 - 8 } & 0.50 & -0.84693 & 0.948535 & -1.12924 & -0.75883 & -1.41323 & -0.30216 \\
\cline { 2 - 8 } & 0.75 & 0.810473 & 0.957611 & -1.08063 & -0.76609 & -1.19391 & -0.30365 \\
\hline
\end{tabular}

Table 4. Missing values and physical quantities with the variation of $K$ and $\theta_{r}$

\begin{tabular}{|c|c|c|c|c|c|c|c|}
\hline$\theta_{r}$ & $\mathrm{~K}$ & $c_{f}$ & $\mathrm{Nu}$ & $f^{\prime \prime}(0)$ & $\theta^{\prime}(0)$ & $h^{\prime}(0)$ & $g^{\prime}(0)$ \\
\hline \multirow{4}{*}{2} & 0.25 & -1.22889 & 0.972569 & -0.98311 & -0.72943 & -1.18624 & -0.23726 \\
\cline { 2 - 8 } & 0.50 & -0.48457 & 0.977167 & -0.96913 & -0.73288 & -1.09908 & -0.23782 \\
\cline { 2 - 8 } & 0.75 & 0.238783 & 0.981313 & -0.95513 & -0.73599 & -1.02391 & -0.23833 \\
\cline { 2 - 8 } & 1.00 & 0.941322 & 0.985133 & -0.94132 & -0.73885 & -0.95846 & -0.2388 \\
\hline \multirow{4}{*}{3} & 0.25 & -0.84788 & 0.953181 & -1.1305 & -0.71489 & -1.41556 & -0.30264 \\
\cline { 2 - 8 } & 0.50 & 0 & 0.957948 & -1.10551 & -0.71846 & -1.29651 & -0.30341 \\
\cline { 2 - 8 } & 0.75 & 0.811233 & 0.962374 & -1.08164 & -0.72178 & -1.19556 & -0.30412 \\
\hline \multirow{4}{*}{4} & 1.00 & 1.588522 & 0.966567 & -1.05902 & -0.72493 & -1.10901 & -0.30481 \\
\cline { 2 - 8 } & 0.25 & -0.69542 & 0.945019 & -1.19215 & -0.70876 & -1.51055 & -0.33355 \\
\cline { 2 - 8 } & 0.50 & 0.193561 & 0.949878 & -1.16136 & -0.71241 & -1.37646 & -0.33442 \\
\cline { 2 - 8 } & 0.75 & 1.038152 & 0.954463 & -1.13253 & -0.71585 & -1.26372 & -0.33525 \\
\hline \multirow{4}{*}{5} & 1.00 & 1.842703 & 0.958862 & -1.10562 & -0.71915 & -1.1678 & -0.33606 \\
\cline { 2 - 8 } & 0.25 & -0.61312 & 0.940517 & -1.22623 & -0.70539 & -1.56285 & -0.35158 \\
\cline { 2 - 8 } & 0.50 & 0.297974 & 0.945431 & -1.1919 & -0.70907 & -1.41993 & -0.35252 \\
\cline { 2 - 8 } & 0.75 & 1.160086 & 0.950114 & -1.16009 & -0.71259 & -1.30042 & -0.35343 \\
\hline
\end{tabular}

Table 5. Comparison of missing values with $M$.

\begin{tabular}{|c|c|c|c|c|c|c|}
\hline \multirow{2}{*}{$M$} & \multicolumn{2}{|c|}{$f^{\prime \prime}(0)$} & \multicolumn{2}{c|}{$\theta^{\prime}(0)$} & \multicolumn{2}{c|}{$h^{\prime}(0)$} \\
\cline { 2 - 7 } & original & new & Original & new & original & new \\
\hline 0.25 & -1.76534 & -1.00498 & -1.36638 & -0.73038 & -0.52405 & -1.18383 \\
\hline 0.5 & -1.86036 & -1.04454 & -1.3471 & -0.72547 & -0.51891 & -1.24028 \\
\hline 0.75 & -1.94925 & -1.08118 & -1.32952 & -0.72106 & -0.51438 & -1.29269 \\
\hline 1 & -2.03303 & -1.11539 & -1.31336 & -0.71707 & -0.51034 & -1.34172 \\
\hline 1.25 & -2.11248 & -1.14752 & -1.2984 & -0.71345 & -0.50671 & -1.38787 \\
\hline 1.5 & -2.18821 & -1.17789 & -1.28448 & -0.71015 & -0.50341 & -1.43154 \\
\hline 1.75 & -2.26069 & -1.20672 & -1.27145 & -0.70714 & -0.50039 & -1.47305 \\
\hline 2 & -2.33031 & -1.23422 & -1.25921 & -0.70437 & -0.49762 & -1.51268 \\
\hline 2.25 & -2.39737 & -1.26053 & -1.24765 & -0.70183 & -0.49506 & -1.55063 \\
\hline 2.5 & -2.46214 & -1.2858 & -1.23671 & -0.69948 & -0.49268 & -1.58709 \\
\hline
\end{tabular}


Numerical Studies on the Effects of Variable Viscosity and Thermal Conductivity with Joules Dissipation on the MHD Micropolar Fluid Flow, Heat and Mass Transfer Past a Stretching Porous Surface Embedded in a Porous Medium

Table 6. Comparison of physical quantities with $M$.

\begin{tabular}{|c|c|c|c|c|}
\hline \multirow{2}{*}{$M$} & \multicolumn{2}{|c|}{$c_{f}$} & \multicolumn{2}{c|}{$N u$} \\
\cline { 2 - 5 } & original & new & original & New \\
\hline 0.25 & -1.05921 & -0.30149 & -1.36638 & 0.973833 \\
\hline 0.5 & -1.11622 & -0.31336 & -1.3471 & 0.967294 \\
\hline 0.75 & -1.16955 & -0.32435 & -1.32952 & 0.961409 \\
\hline 1 & -1.21982 & -0.33462 & -1.31336 & 0.956091 \\
\hline 1.25 & -1.26749 & -0.34426 & -1.2984 & 0.94687 \\
\hline 1.5 & -1.31293 & -0.35337 & -1.28448 & 0.942852 \\
\hline 1.75 & -1.35642 & -0.36202 & -1.27145 & 0.939165 \\
\hline 2 & -1.39818 & -0.37026 & -1.25921 & 0.935772 \\
\hline 2.25 & -1.43842 & -0.37816 & -1.24765 & 0.93264 \\
\hline 2.5 & -1.47729 & -1.23671 & -1.23671 & \\
\hline
\end{tabular}

\section{CONCLUSiON}

In this study the effects of variable viscosity and thermal conductivity with Joules dissipation on MHD micropolar fluid flow, heat and mass transfer past a stretching porous surface embedded in a porous medium is investigated. It is observed from the above discussions that the viscosity and thermal conductivity parameters along with the other parameters mentioned above have significant effects on velocity, temperature, concentration and micro-rotation distributions within the boundary layer. Also the presence of micro-constituents results significant change of behaviours in the flow problems in micropolar fluid in comparison with the ordinary fluids. The following conclusions can be drawn from this study:

1. Velocity decreases for the increase of both viscosity and magnetic parameter.

2. Microrotation increases with the increase of both viscosity and magnetic parameter.

3. Temperature increases for the increase of viscosity and magnetic parameter while it decreases with thermal conductivity.

4. Skin friction increases with the increase of both viscosity and thermal conductivity.

5. Nusselt number decreases with the increase of both viscosity and thermal conductivity.

This investigation must be helpful for further study of micropolar fluid in different branches of fluid dynamics.

\section{Nomenclatures}

$\beta=$ Volumetric coefficient of thermal expansion

$\gamma=$ Spin-gradient or micro rotation viscosity

$\eta=$ Dimensionless co-ordinates

$\lambda=$ Thermal conductivity

$\lambda_{\infty}=$ Thermal conductivity of the ambient fluid

$\mu=$ Dynamic viscosity

$\mu_{\infty}=$ Dynamic viscosity of the ambient fluid

$v=$ Kinematic viscosity

$\kappa=$ Vortex viscosity

$\rho=$ Density

$\sigma=$ Electrical conductivity

$\theta=$ Dimensionless temperature

$\theta_{c}=$ Dimensionless reference temperature corresponding to thermal conductivity parameter 
Numerical Studies on the Effects of Variable Viscosity and Thermal Conductivity with Joules Dissipation on the MHD Micropolar Fluid Flow, Heat and Mass Transfer Past a Stretching Porous Surface Embedded in a Porous Medium

$\theta_{r}=$ Dimensionless reference temperature corresponding to viscosity parameter

$\tau_{\mathrm{w}}=$ Wall shear stress

$\mathrm{q}_{\mathrm{w}}=$ Heat transfer from the plate

$c_{\mathrm{p}}=$ Specific heat at constant pressure

$u=$ Velocity in the $x$-direction

$v=$ Velocity in the $y$-direction

$f^{\prime}=$ Dimensionless velocity

$h=$ Dimensionless micro-rotation

$g=$ Dimensionless species concentration

$C=$ Species concentration

$T=$ Temperature

$T_{\infty}=$ Ambient temperature

$T_{w}=$ Wall temperature

$C_{w}=$ Species concentration at the wall

$C_{\infty}=$ Species concentration far from the wall

$U_{w}=$ Stretching velocity

$j=$ Micro rotation density

$D=$ Mass diffusivity

$B_{0}=$ Strength of the magnetic field

$S_{c}=\frac{v_{\infty}}{D}$, Schmidt number

$P_{r}=\frac{v_{\infty} \rho c_{\mathrm{p}}}{\lambda_{\infty}}$, Prandtl number

$E_{c}=\frac{U_{w}^{2}}{c_{p}\left(T_{w}-T_{\infty}\right)}$, Eckert number.

$R e_{x}=\frac{U_{w} x}{v_{\infty}}$, Local Reynolds number.

$M=\frac{2 \sigma B_{0}^{2} x}{\rho(1+\mathrm{m}) \mathrm{U}_{\mathrm{w}}}$, Magnetic parameter (Hartmann number)

$K=\frac{\kappa}{\rho v_{\infty}}$, coupling constant parameter

$K_{l}=\frac{K}{\mathrm{i}}$, coupling parameter.

$K_{p}=$ Permeability of the medium.

$R_{1}=$ Permeability parameter.

$N u=$ Nusselt number

$N u_{x}=$ Local Nusselt number

$c_{f}=$ Skin - friction coefficient

\section{Subscripts}

$\mathbf{w}$, the condition at the wall

$\infty$, the condition far away from the surface

\section{Superscripts}

', Differentiation with respect to $\eta$ 
Numerical Studies on the Effects of Variable Viscosity and Thermal Conductivity with Joules Dissipation on the MHD Micropolar Fluid Flow, Heat and Mass Transfer Past a Stretching Porous Surface Embedded in a Porous Medium

\section{REFERENCES}

[1] Eringen A.C., Simple micro fluids, Int. J. Eng. Sci., 2, 205-217, 1964.

[2] Eringen A.C., Theory of micropolar fluids J. Math. Mech., 16, 1-18, 1966.

[3] Eringen A.C., Theory of micropolar fluids J. Math. Anal. Appl., 38, 480-496, 1972.

[4] Guram G.S., Smith A.C., Stagnation flow of micropolar fluids with strong and weak interactions, Comp. Math. With Appl., 6, 213-233, 1980.

[5] Ishak A., Nazar R., Pop I., Heat transfer over a stretching surface with variable heat flux in micropolar fluids, Phys. Letter A., 372, 559-561, 2008.

[6] Thakur P.M., Hazarika G. C., Effects of variable viscosity and thermal conductivity on the MHD flow of micropolar fluid past an accelerated infinite vertical insulated plate, Int. J. of Heat and Tech. USA., 33, 7378, 2015.

[7] Abel M.S., Mahesha N., Effects of thermal buoyancy and variable thermal conductivity in a power law fluid past a vertical stretching sheet in the presence of non uniform heat source, Int. J. Nonlinear Mech., 44, 1-12, 2009.

[8] Chen C.H., Effect of viscous dissipation on Heat Transfer in a non-Newtonian liquid film over an unsteady stretching sheet, J. of non-Newtonian Fluid Mech., 135, 128-135, 2006.

[9] Hazarika G.C., Hazarika S., Effects of Variable Viscosity and Thermal conductivity on Magnetohydrodynamics mixed Convective Flow over a Stretching Surface with Radiation, Int. J. of Sci. Research Eng. Tech., 4, 809-815, 2015.

[10] Kelson N.A., Desseaux A., Effects of surface conditions on flow of micropolar fluid driven by a porous stretching sheet, Int J. Eng. Sci., 39, 1881-1897, 2001.

[11] Khound P.K., Hazarika G.C., The effect of variable viscosity and thermal conductivity on liquid film on an unsteady stretching surface, Proc. of $46^{\text {th }}$ Annual Tech. Session, Ass Sc. Soc., 47-56, 2000.

[12] Vajravelu K., Hadjinicalaou A., Convective heat transfer in an electrically conducting fluid at a stretching surface in uniform free stream, Int. J. Eng. Sci., 35, 1237-1244, 1997.

[13] Ahmed N., Hazarika G.C., MHD free and forced convection and mass transfer flow past a porous vertical plate, Int. J. of Heat and Tech., 30, 97-106, 2012.

[14] Sarma U., Hazarika G. C., Effects of variable viscosity and thermal conductivity on heat and mass transfer flow along a vertical plate in the presence magnetic field, Int. Int. J. Phys. Education., 5, 1-7, 2011.

[15] Hazarika G.C., Phukan B.,Effects of variable viscosity and thermal conductivity on magnetohydrodynamic free convection flow of a micropolar fluid past a stretching plate through porous medium with radiation, heat generation, and Joules dissipation. Turk J. Phys., 40, 40-51, 2016.

[16] Anjali Devi S.P., Ganga B., Effects of viscous and Joules dissipation on MHD flow, heat and mass transfer past a stretching porous surface embedded in a porous medium, Nonlinear Anal.: Modelling and Control, $14,303-314,2009$.

[17] Lai F.C., Kulacki F.A., The effect of variable viscosity on convective heat and mass transfer along a vertical surface in saturated porous medium, Int. J. Heat Mass Tran., 33, 1028-1031, 1990. 
Numerical Studies on the Effects of Variable Viscosity and Thermal Conductivity with Joules Dissipation on the MHD Micropolar Fluid Flow, Heat and Mass Transfer Past a Stretching Porous Surface Embedded in a Porous Medium

\section{AUTHORS' BIOGRAPHY}

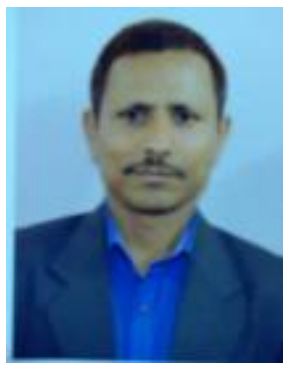

Surajit Dutta, Designation: Assistant Professor. Educational Qualification:

M.Sc. (Mathematics), from Dibrugarh University,Dibrugarh, Assam, India. Now doing research in the field of micropolar fluid dynamics under the supervision of Professor G.C. Hazarika of Dibrugarh University.

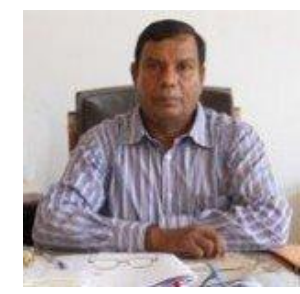

Prof.Gopal Chandra Hazarika, Educational Qualification: M.Sc., Ph.D. Gold Medal in M.Sc. Life Member of various organizations like Assam Academy of Mathematics, Indian Mathematics Association etc.. More than twenty Scholars got $\mathrm{Ph} . \mathrm{D}$. under his supervision. More than one hundred research articles published in various national and international journals. Visited various colleges and Universities of India as member of NAAC peer team. Associated with many social and cultural organizations.

Citation: S. Dutta, G. C. Hazarika, "Numerical Studies on the Effects of Variable Viscosity and Thermal Conductivity with Joules Dissipation on the MHD Micropolar Fluid Flow, Heat and Mass Transfer Past a Stretching Porous Surface Embedded in a Porous Medium ", International Journal of Scientific and Innovative Mathematical Research, vol. 6, no. 2, p. 1-16, 2018., http://dx.doi.org/10.20431/23473142.0602001

Copyright: $\odot 2018$ Authors. This is an open-access article distributed under the terms of the Creative Commons Attribution License, which permits unrestricted use, distribution, and reproduction in any medium, provided the original author and source are credited. 